\title{
FGF21 protects against ox-LDL induced apoptosis through suppressing CHOP expression in THP1 macrophage derived foam cells
}

\author{
En Li ${ }^{1,2}$, Ting Wang ${ }^{3}$, Feng Wang ${ }^{4}$, Tao Wang ${ }^{5}$, Li-qiang Sun², Li Li ${ }^{2}$ Shao-hui Niu² and Jin-ying Zhang ${ }^{\text {** }}$
}

\begin{abstract}
Background: FGF21, as a member of the fibroblast growth factor superfamily, is an important endogenous regulator to systemic glucose and lipid metabolism. Elevated serum FGF21 levels have been reported in subjects with coronary heart disease and carotid artery plaques. The formation and apoptosis of foam cell, induced by ox-LDL and oxysterols, are key steps in the development of atherosclerosis.

Methods: In this study, THP1 derived macrophages were induced into foam cells by ox-LDL or sterols. The formation and apoptosis of foam cells treated with or without FGF21 were analyzed.

Results: We demonstrated that the accumulation of cholesterol was decreased after FGF21 treatment in THP1 macrophage derived foam cells. Consistently, the apoptosis of macrophage was alleviated dramatically with FGF21 treatment. ERK1/2 knockdown didn't abrogate the effect of FGF21 on THP1 macrophage derived foam cells. However, FGF21 suppressed the induced expression of CHOP and DR5 in THP1 macrophage derived foam cells.

Conclusion: FGF21 protects against the formation and apoptosis of THP1 macrophages derived foam cells through suppressing the expression of CHOP.
\end{abstract}

Keywords: Macrophage, ER stress, FGF21, Foam cell, CHOP

\section{Background}

Fibroblast growth factor (FGF) superfamily, has an important functions in metabolic processes. Among members of the FGF superfamily, FGF21 plays a very important regulatory role in glucose and lipid homeostasis [1-3].

The role of FGF21 in glucose and lipid metabolism has been well studied $[4,5]$. Human recombinant FGF21 has been demonstrated to stimulate glucose incorporation in mouse and human adipocytes, and to lower blood glucose and triglyceride levels in diabetic and obese mice as well as diabetic monkeys [6]. By contrast, FGF21 deficient mice showed mild weight gain, slightly impaired glucose homeostasis, and also developed hepatosteatosis and obvious impairments in ketogenesis and glucose control when raised on a ketogenic diet [7]. These findings suggest that FGF21 is an important metabolic hormone in maintaining

\footnotetext{
* Correspondence: jyzhangzzu@126.com

1 Department of Cardiovascular internal medicine, The first Affiliated Hospital of Zhengzhou University, Zhengzhou, P. R. China

Full list of author information is available at the end of the article
}

glucose and lipid homeostasis. Recently, it is reported that serum FGF21 levels are increased in coronary heart disease (CHD) and FGF21 is also found in carotid artery plaques $[5,8,9]$. In myocardial infarction, FGF21could attenuate pathological myocardial remodeling through the adiponectin-dependent mechanism [10]. What's more, FGF21 have also been found to attenuate hyperlipidemia and diabetes induced early-stage apoptosis [11]. Based on these results, FGF21 has been proposed to be associated with arteriosclerosis. However, the role of FGF21 in arteriosclerosis remains unclear.

In normal macrophages, low density lipoprotein (LDL) cholesterol particles are loaded from late endosomes to the ER. In the ER, cholesterol is esterified and accumulated to form inert lipid droplets $[12,13]$. In atherosclerotic macrophages, ER-mediated cholesterol reesterification is markedly impaired resulting in excessive intracellular deposits of nonesterified cholesterol and the formaition of foam cells [13], where intraluminal ER oxidoreductases oxidize cholesterol to 7-ketocholesterol (7-KC) and other oxysterols. 
Oxysterols are highly cytotoxic and may induce cell death through ROS-mediated oxidative damage [14]. Prolonged ER stress contributes to apoptosis of lesional macrophages, which is associated with robust expression of C/EBP homologous protein (CHOP) in human lesions [15] and atherosclerotic plaques of apolipoprotein (apo) E-deficient mice [16]. Inactivating $\mathrm{CHOP}$ in apoE-deficient mice slows down macrophage apoptosis and plaque necrosis [17-19]. CHOP contributes to ER stress-induced macrophage death by inducing Fas activation, depletion of ER-associated calcium stores, and release of apoptogens from mitochondria [19]. Moreover, CHOP is found to induce cell apoptosis through activating death receptor 5 (DR5) in human carcinoma cells [20].

\section{Methods}

Isolation and oxidation of Low density lipoprotein

The native low-density lipoprotein (LDL) was obtained from Sigma. LDL was oxidized with $\mathrm{CuSO}_{4}$ at $37{ }^{\circ} \mathrm{C}$ for $18 \mathrm{~h}$ and transferred into ethylene diamine tetraacetic acid (EDTA; $200 \mathrm{mM}$ ) in phosphate-buffered saline (PBS) for $24 \mathrm{~h}$ at $4{ }^{\circ} \mathrm{C}$ to remove $\mathrm{Cu}^{2+}$. Subsequently, the product was dialyzed in PBS for $24 \mathrm{~h}$ at $4{ }^{\circ} \mathrm{C}$ to remove EDTA. LDL oxidation was confirmed by thiobarbituric acid reaction substances with malondialdehyde as the standard. The content of ox-LDL was 1.12 compared with $0.30 \mathrm{nmol} / 100 \mathrm{mg}$ protein in the native LDL preparation $(\mathrm{p}<0.01)$. The ox-LDL was then sterilized by filtration and stored at $4{ }^{\circ} \mathrm{C}$ as previously described [21].

\section{Cell Culture}

The human THP-1 cells were obtained from the Type Culture Collection of the Chinese Academy of Sciences (Shanghai, China). THP-1 cells were cultured in Roswell Park Memorial Institute medium 1640 (RPMI 1640, Hyclone) containing $10 \%$ fetal bovine serum and $2 \mathrm{mM} \mathrm{L}$-glutamine. The cells were differentiated into macrophages by adding $100 \mathrm{ng} / \mathrm{mL}$ phorbol 12 myristate-13-acetate for $24 \mathrm{~h}$, and the medium was then replaced with that containing ox-LDL $(50 \mathrm{mg} / \mathrm{mL})$ and human FGF21 (Peprotech, $20 \mathrm{nmol} / \mathrm{L}$ ) for $24 \mathrm{~h}$ to obtain fully differentiated foam cells before use in experiments. And human FGF21 (Peprotech, $20 \mathrm{nmol} / \mathrm{L}$ ) was added to cotreat the THP1 macrophage derived foam cell.

\section{SiRNA transfection}

THP-1 cells were transfected with specific siRNA oligomers directed against Erk ( $80 \mathrm{nM}$ ) using Lipofectaminqe 2000 transfection reagent (Invitrogen, Carlsbad, CA) according to the manufacturer's instructions. Negative control siRNA oligomers were used as a negative control. After transfection for $48 \mathrm{~h}$, the cells were exposed to ox-LDL $(50 \mathrm{mg} / \mathrm{L})$ for $24 \mathrm{~h}$. The silencing of target genes was validated by western blotting.

\section{Western blotting}

Total proteins and nuclear proteins from cells were extracted using RIPA lysis buffer and nuclear extraction kits, respectively, following the manufacturer's instructions. Equal amounts of protein were separated by SDS-PAGE and probed with various primary antibodies as indicated. Immunoblots were visualized using ECL reagent, and the integrated optical density (IOD) of immunoreactive bands was measured using Image-Pro Plus software and normalized by house-keeping protein (GAPDH).

\section{Quantitative real-time PCR}

Total RNA was extracted using Trizol reagent (Invitrogen). $2 \mu \mathrm{g}$ of total RNA was reversely transcripted using MMLV Reverse Transcriptase (Invitrogen). Real-time PCR was performed on a Rotor-Gene Q real-time PCR cycler (Roche, Shanghai, China) using SYBR-green PCR master mix kits. The data were analyzed using the Rotor-Gene $\mathrm{Q}$ software (version 1.7, Qiagen), and relative mRNA levels were calculated using the $2^{-\Delta \Delta \mathrm{Ct}}$ method and normalized against 18S rRNA. The primers used for real-time PCR were synthesized by Sangon Biotech (Shanghai, China).

\section{Statistical analyses}

Analysis of variance was conducted to examine whether significant $(P, 0.05)$ main treatment and time effects occurred. Additional post hoc comparisons of treatment means were conducted by using the Dunnett's $t$-test (treatments vs. controls) and Bonferroni $t$-test (selected comparisons) as indicated. Data given represent meansstandard deviation.

\section{Results}

FGF21 decreases the formation of foam cell fromTHP1 derived macrophages

With the development of atherosclerosis, the infiltrated macrophages engulf LDL leading to the accumulation of cholesterol in the droplets, and the formation of foam cells. The uptake of ox-LDL by macrophages is the event that triggers the formation of lipid-laden foam cells. The oil red O-staining (Fig. 1a and b) and intracellular TC quantitative assay (Fig. 1c) indicated that lipid content in differentiated THP-1 cells was significantly increased by ox-LDL, indicating the formation of foam cells. When the cells were co-treated with FGF21, the lipid content decreased dramatically as shown by the reduced lipid droplets (Fig. 1a and b) and lower intracellular TC (Fig. 1c). FACS analysis also demonstrated that ox-LDL induced foam cells decreased dramatically after FGF21 treatment (Fig. 1d). These results show that FGF21 can 


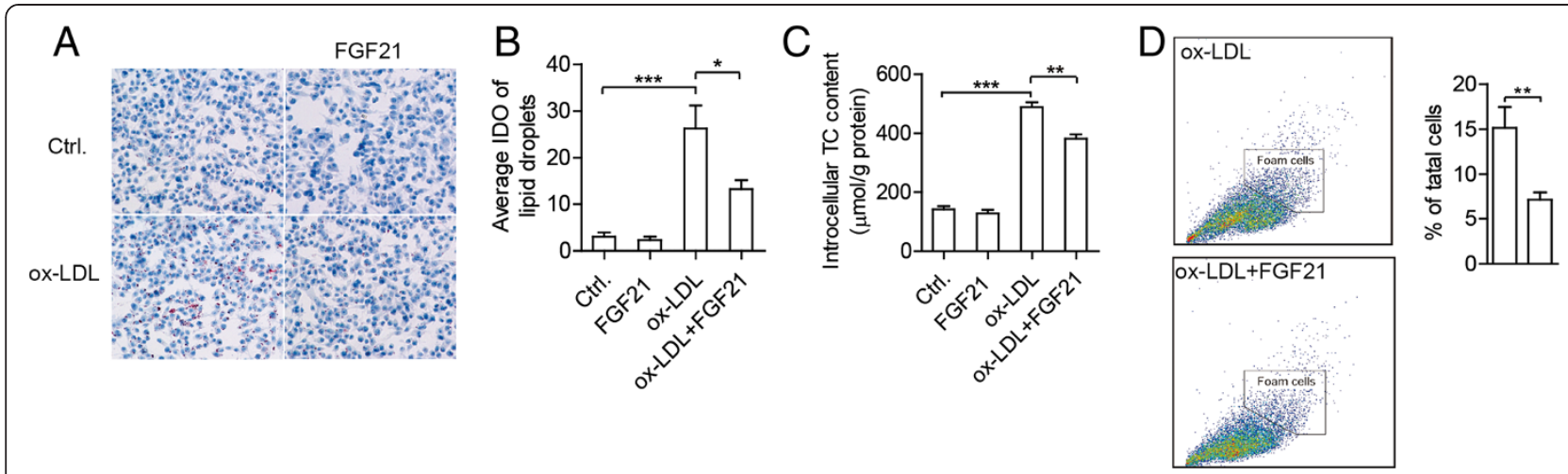

Fig. 1 FGF21 decreased foam cell formation of THP1 derived macrophages. a Representative images and (b) the average integrated optical density $(I O D)$ of lipid droplets stained with oil red $\mathrm{O}$ from differentiated macrophages treated by $50 \mathrm{mg} / \mathrm{L}$ ox-LDL in the presence or absence of $20 \mathrm{nmol} / \mathrm{L}$ FGF21 for $24 \mathrm{~h}$. c The intracellular total cholesterol (TC) content was measured under the same conditions as in a. $\mathbf{d}$ Foam cells were identified by FACS assay, representative images of FACS (left) and the percentages of apoptosis cells (right). Data are presented as the mean \pm S.E. of at least four independent experiments. ${ }^{*}, p<0.05,{ }^{*}, p<0.01$

decrease the accumulation of cholesterol and inhibit foam cell formation in macrophages treated by ox-LDL.

\section{FGF21 protects against ox-LDL and 7-KC induced macrophage apoptosis}

The apoptosis of foam cells duo to over-loaded cholesterol is the key event during the development of atherosclerosis,. We wondered about the effect of FGF21 on the apoptosis of macrophages induced by ox-LDL. As demonstrated by FACS analysis results, the apoptosis of THP1 derived macrophages increased significantly after the treatment of ox-LDL (Fig. 2a and b). Consist with the inhibitory effect of FGF21 on foam cell formation, the apoptosis of THP1 derived macrophages induced by the uptake of ox-LDL decreased dramatically when the cells were treated by FGF21 (Fig. $2 a$ and b). Ox-LDL is composed of many potentially proatherogenic molecules. 7 -ketocholesterol $(7-\mathrm{KC})$, as one of the oxysterols, is found in relatively large abundance in ox-LDL, as well as in atherosclerotic plaque and foam cells in vivo. We wondered if FGF21 had the same effect on 7-KC induced apoptosis of macrophages. As the results of FACS analysis, FGF21 inhibited significantly the 7-KC induced apoptosis of THP1 derived macrophages (Fig. $2 \mathrm{c}$ and d). These results revealed that FGF21 protected against ox-LDL and oxysterols induced macrophage apoptosis.

\section{The effect of FGF21 on macrophages is independent upon ERK signaling pathway}

ERK signaling pathway plays significant role in FGF21 function $[20,22]$. To test if the inhibition function of FGF21 on foam cell formation and apoptosis of macrophages is ERK pathway dependent, siRNA selectively targeted ERK $1 / 2$ were used to knocked down the protein in macrophages. Western blotting results showed that ERK1/2 protein levels decreased dramatically after targeted siRNA transfection in macrophages (Fig. 3a). As shown in Fig. 3b, in compare with ox-LDL treatment group, FGF-21 alleviated

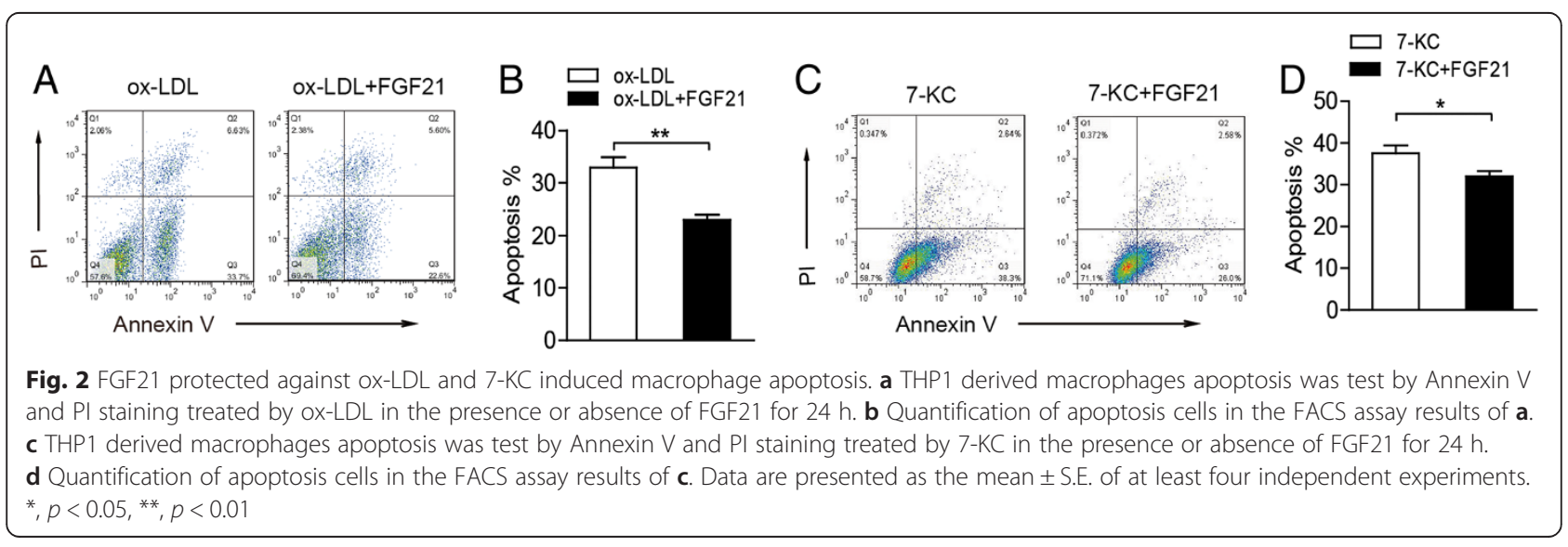




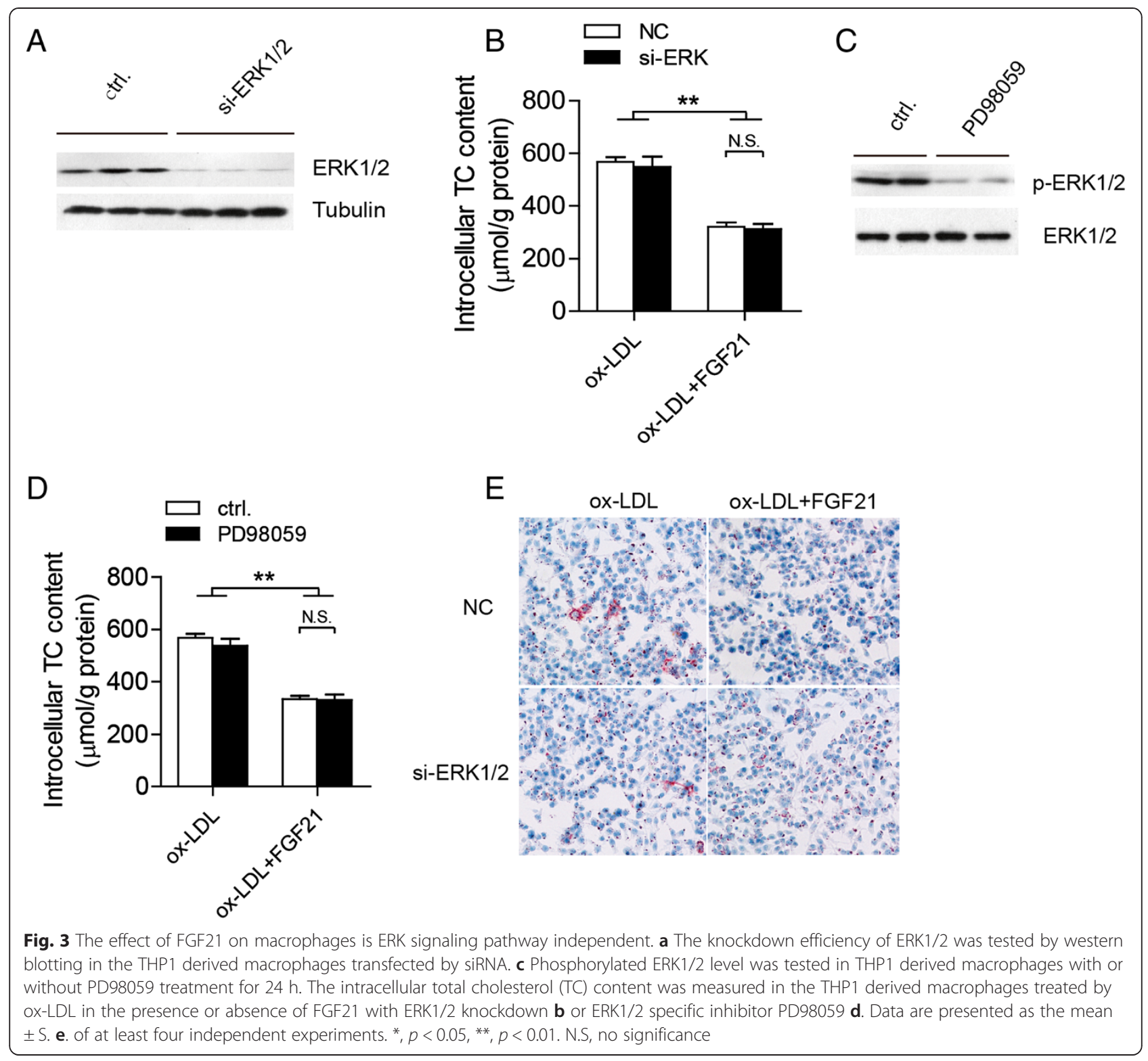

cholesterol accumulation in both nc and si-ERK1/2 transfected cells, however, the less of ERK $1 / 2$ had no significantly effect on lipid cholesterol accumulation and foam cell formation in THP1 derived macrophages (Fig. 3b and e). To further confirm that ERK1/2 signaling pathway is not involved, we treated THP1 macrophage derived foam cells with PD98059, a phosphorylated ERK1/2 specific inhibitor. With the addition of PD98059, ERK signaling pathway was almost blocked as the decreased phosphorylated ERK1/2 levels (Fig. 3c). But the cholesterol levels remained the same as that in the control group with or without FGF21 treatment (Fig. 3d) These results demonstrate that the functions of FGF21 on THP1 derived foam cells are ERK signaling pathway independent.
FGF21 suppresses CHOP expression induced by ox-LDL and 7-KC in macrophages

The apoptosis of macrophages induced by ox-LDL and oxysterols is considered through the activated the Unfolded Protein Response pathway (UPR pathway), and CHOP, the important component in UPR pathway, is considered as the key player. Interestingly, when we tested the RNA and protein levels of CHOP in THP1 derived macrophages, we found that after FGF21 treatment, ox-LDL induced RNA and protein levels of CHOP, were deceased dramatically (Fig. 4a-c). The apoptosis of macrophages induced by 7-KC was also considered to be caused by the upregulation of CHOP. In our data (Fig. 4b-c), FGF21 also suppressed dramatically 7-KC induced CHOP 


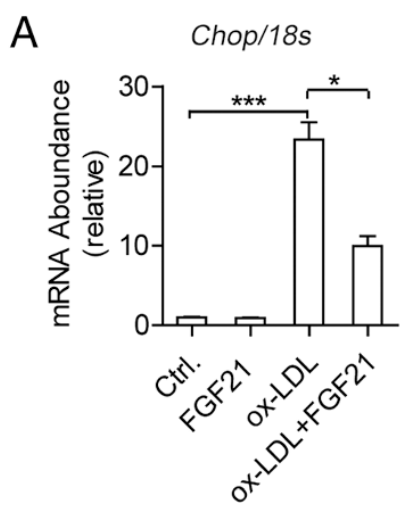

C

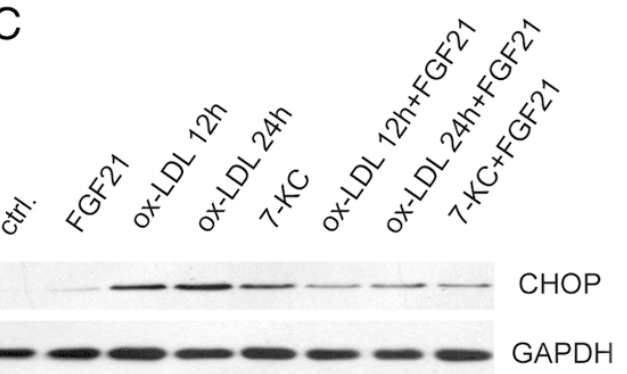

$\mathrm{E}$

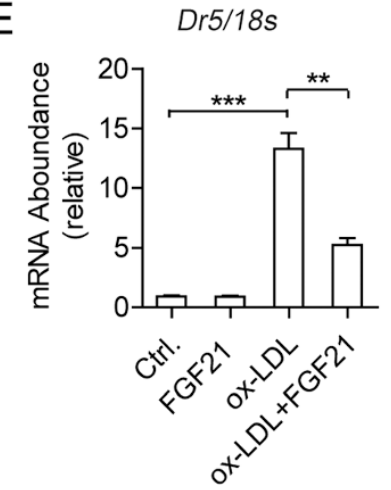

B

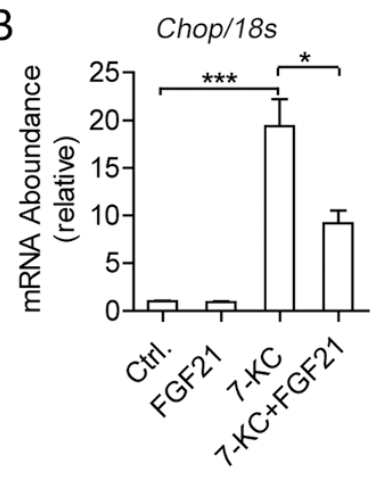

D

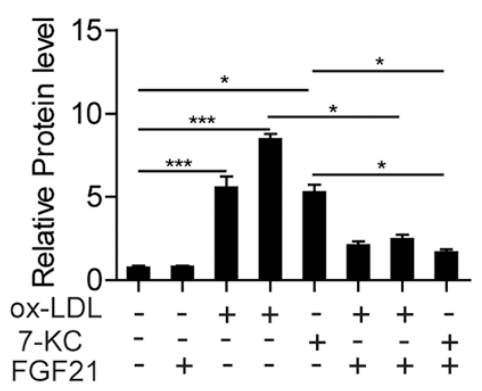

$\mathrm{F}$

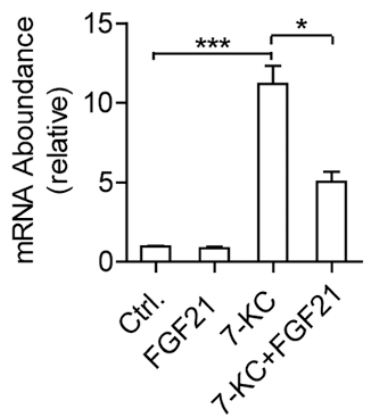

Fig. 4 FGF21 suppressed CHOP expression induced by ox-LDL and 7-KC in macrophages. CHOP mRNA level was tested by Real-time PCR in THP1 derived macrophages treated by ox-LDL (a) or 7-KC (b) in the presence or absence of FGF21 for $24 \mathrm{~h}$, and $18 \mathrm{~S}$ rRNA was used as internal control. c CHOP protein level was tested by western blotting. $\mathbf{d}$ Quantification of the results in c. DR5 mRNA level was measured by Real-time PCR in THP1 derived macrophages treated by ox-LDL (e) or 7-KC (f) in the presence or absence of FGF21. 18S rRNA was used as internal control. Data are presented as the mean \pm S.E. of at least three independent experiments. ${ }^{*}, p<0.05,{ }^{*}, p<0.01$. N.S, no significance

expression. Additionally, Dearth Receptor 5 (DR5), the direct downstream target of $\mathrm{CHOP}$ and the main mediator in CHOP-mediated cell apoptosis, decreased significantly after FGF21 treatment in THP1 derived macrophages (Fig. 4c-d).

To investigate whether the reduction of $\mathrm{CHOP}$ is the major reason of FGF21 protection effects, we introduced siRNA specifically targeted $\mathrm{CHOP}$ to knockdown $\mathrm{CHOP}$ in THP1 derived macrophages. After confirmation of decreased CHOP levels as shown in Fig. 5a, the expression level of DR5 was downregulated in both oxLDL and 7-KC treated macrophages (Fig. 5b-c). With the inhibition on CHOP signaling, the apoptosis of macrophages induced by both oxLDL and 7-KC were dramatically decreased after si-CHOP transfection (Fig. 5d-e). However, the protection of FGF21 on macrophage apoptosis was almost abolished in macrophages when $\mathrm{CHOP}$ was knockdown (Fig. 5d-e). 


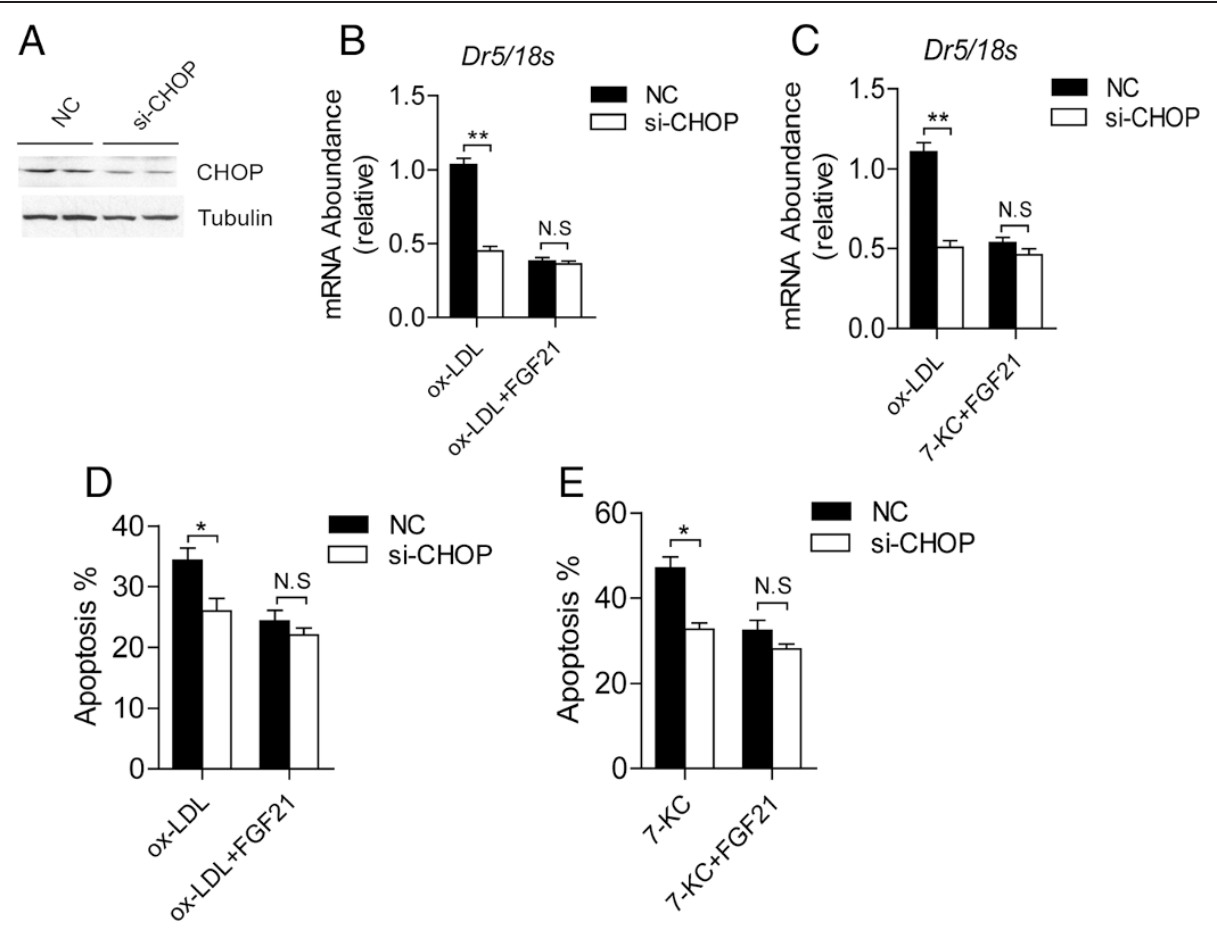

Fig. 5 The protection effects of FGF21 on apoptosis were through suppress CHOP signaling in macrophages. a The knockdown efficiency of CHOP was tested by western blotting in the THP1 derived macrophages transfected by siRNA. DR5 mRNA level was measured by Real-time PCR in THP1 derived macrophages with si-CHOP transfection treated by ox-LDL (b) or 7-KC (C) in the presence or absence of FGF21. 18S rRNA was used as internal control. d-e Quantification of apoptosis cells in FACS assay of THP1 cells treated as indicated. Data are presented as the mean \pm S.E. of at least three independent experiments. ${ }^{*}, p<0.05,{ }^{* *}, p<0.01$. N.S, no significance

So, the conclusion is that the protection effect of FGF21 on macrophage apoptosis is through CHOP pathway suppression.

\section{Discussion and conclusion}

FGF21, as a member of hormone-like subgroup within the FGF superfamily, is emerging as a key regulator of energy homeostasis and a potential target for the treatment of diabetes, cardiovascular disease, and obesity [4, 8, 23]. A previous study showed that incubation of rodent cardiac microvascular endothelial cells (CMECs) with oxidized LDL led to increased FGF21 expression and inhibited CMEC apoptosis [24]. These results supported the hypothesis that FGF21 function as an endogenous protective factor in the cardiovascular system can improve endothelial function during early stages of atherosclerosis. Moreover, elevated serum FGF21 levels have recently been reported in patients with CHD and are associated with the presence of carotid artery plaques $[5,8]$. However, the role of FGF21 in atherosclerosis plaques remains unclear. In this study, we present that FGF21 can protect macrophage against foam cell formation and apoptosis.

In summary, FGF21 repressed the cholesterol accumulation and foam cell formation in THP1 derived macrophage induced by ox-LDL or 7-KC. And the apoptosis of foam cell was decreased with the treatment of FGF21. Moreover,the effect of FGF21 on THP1 derived macrophages was ERK1/2 MAPK pathway independent,and was mediated through suppressing the expression of CHOP and its downstream target DR5. These findings provide evidence for the role of FGF21 in arteriosclerosis and present the new direction for atherosclerosis prevention.

\section{Competing interests}

The authors declare that they have no competing interests.

\section{Authors' contributions}

EL was involved in the conception, design of the study, doing the experiment, the analysis and interpretation of raw data and writing the article. TW participated in the design of the study, performed data entry, parts of the statistical analysis and helped to draft the manuscript. FW and JYZ participated in the design of the study, reviewed and edited the manuscript. TW, LQS, LL and SHN were substantially participated in doing the experiment and critical revision of the article prior to submission. All authors read and approved the final manuscript.

\section{Acknowledgements}

This work was supported by grants from science and technology projects in Henan province (No. 142102310109 and 122102310088). We are grateful to Prof. Zongfang Liu and Prof. Lihua Zhang for helpful discussions and technical assistance. We thank Prof. Jian Liguo for helpful comments.

\section{Author details}

'Department of Cardiovascular internal medicine, The first Affiliated Hospital of Zhengzhou University, Zhengzhou, P. R. China. ${ }^{2}$ Department of Cardiovascular internal medicine, The second Affiliated Hospital of 
Zhengzhou University, Zhengzhou, P. R. China. ${ }^{3}$ Department of Gerontology, Shaanxi People's Hospital, Xi'an, P. R. China. ${ }^{4}$ CAS Key Laboratory of Computational Biology, CAS-MPG Partner Institute for Computational Biology, Shanghai Institutes for Biological Sciences, Chinese Academy of Sciences, Shanghai, P. R. China. ${ }^{5}$ Department of Gerontology, The second Affiliated Hospital of Zhengzhou University, Zhengzhou, P. R. China.

Received: 10 October 2014 Accepted: 24 July 2015

Published online: 30 July 2015

\section{References}

1. Cuevas-Ramos D, Aguilar-Salinas CA, Gomez-Perez FJ. Metabolic actions of fibroblast growth factor 21. Curr Opin Pediatr. 2012;24(4):523-9.

2. Ding X, Boney-Montoya J, Owen BM, Bookout AL, Coate KC, Mangelsdorf $D$ J, et al. betaklotho is required for fibroblast growth factor 21 effects on growth and metabolism. Cell Metab. 2012;16(3):387-93.

3. Woo YC, Xu A, Wang Y, Lam KS. Fibroblast growth factor 21 as an emerging metabolic regulator: clinical perspectives. Clin Endocrinol (Oxf). 2013;78(4):489-96

4. Habegger KM, Stemmer K, Cheng C, Muller TD, Heppner KM, Ottaway N, et al. Fibroblast growth factor 21 mediates specific glucagon actions. Diabetes. 2013;62(5):1453-63.

5. Lin Z, Wu Z, Yin X, Liu Y, Yan X, Lin S, et al. Serum levels of FGF-21 are increased in coronary heart disease patients and are independently associated with adverse lipid profile. PLoS One. 2010;5(12), e15534.

6. Kharitonenkov A, Wroblewski VJ, Koester A, Chen YF, Clutinger CK, Tigno XT, et al. The metabolic state of diabetic monkeys is regulated by fibroblast growth factor-21. Endocrinology. 2007;148(2):774-81.

7. Badman MK, Koester A, Flier JS, Kharitonenkov A, Maratos-Flier E. Fibroblast growth factor 21-deficient mice demonstrate impaired adaptation to ketosis. Endocrinology. 2009;150(11):4931-40.

8. An SY, Lee MS, Yi SA, Ha ES, Han SJ, Kim HJ, et al. Serum fibroblast growth factor 21 was elevated in subjects with type 2 diabetes mellitus and was associated with the presence of carotid artery plaques. Diabetes Res Clin Pract. 2012;96(2):196-203.

9. Li G, Gu HM, Zhang DW. ATP-binding cassette transporters and cholesterol translocation. IUBMB Life. 2013;65(6):505-12.

10. Joki Y, Ohashi K, Yuasa D, Shibata R, Ito M, Matsuo K, et al. FGF21 attenuates pathological myocardial remodeling following myocardial infarction through the adiponectin-dependent mechanism. Biochem Biophys Res Commun. 2015:459(1):124-30

11. Zhang C, Shao M, Yang H, Chen L, Yu L, Cong W, et al. Attenuation of hyperlipidemia- and diabetes-induced early-stage apoptosis and latestage renal dysfunction via administration of fibroblast growth factor-21 is associated with suppression of renal inflammation. PLoS One. 2013:8(12), e82275

12. Brown MS, Goldstein JL. Lipoprotein metabolism in the macrophage: implications for cholesterol deposition in atherosclerosis. Annu Rev Biochem. 1983:52:223-61.

13. Maxfield FR, Tabas I. Role of cholesterol and lipid organization in disease. Nature. 2005;438(7068):612-21.

14. Feng B, Yao PM, Li Y, Devlin CM, Zhang D, Harding HP, et al. The endoplasmic reticulum is the site of cholesterol-induced cytotoxicity in macrophages. Nat Cell Biol. 2003;5(9):781-92.

15. Myoishi M, Hao H, Minamino T, Watanabe K, Nishihira K, Hatakeyama K, et al. Increased endoplasmic reticulum stress in atherosclerotic plaques associated with acute coronary syndrome. Circulation. 2007;116(11):1226-33.

16. Zhou J, Lhotak S, Hilditch BA, Austin RC. Activation of the unfolded protein response occurs at all stages of atherosclerotic lesion development in apolipoprotein E-deficient mice. Circulation. 2005;111(14):1814-21.

17. Thorp E, Li G, Seimon TA, Kuriakose G, Ron D, Tabas I. Reduced apoptosis and plaque necrosis in advanced atherosclerotic lesions of Apoe-/- and Ldlr-/- mice lacking CHOP. Cell Metab. 2009;9(5):474-81.

18. Tsukano H, Gotoh T, Endo M, Miyata K, Tazume H, Kadomatsu T, et al. The endoplasmic reticulum stress-C/EBP homologous protein pathway-mediated apoptosis in macrophages contributes to the instability of atherosclerotic plaques. Arterioscler Thromb Vasc Biol. 2010;30(10):1925-32

19. Ozcan L, Tabas I. Pivotal role of calcium/calmodulin-dependent protein kinase II in ER stress-induced apoptosis. Cell Cycle. 2010;9(2):223-4.
20. Fisher FM, Kleiner S, Douris N, Fox EC, Mepani RJ, Verdeguer F, et al. FGF21 regulates $P G C-1$ alpha and browning of white adipose tissues in adaptive thermogenesis. Genes Dev. 2012;26(3):271-81.

21. Hu YW, Wang Q, Ma X, Li XX, Liu XH, Xiao J, et al. TGF-beta1 up-regulates expression of $A B C A 1, A B C G 1$ and $S R-B 1$ through liver $X$ receptor alpha signaling pathway in THP-1 macrophage-derived foam cells. J Atheroscler Thromb. 2010;17(5):493-502.

22. Lu P, Yan J, Liu K, Garbacz WG, Wang P, Xu M, et al. Activation of aryl hydrocarbon receptor dissociates fatty liver from insulin resistance by inducing fibroblast growth factor 21. Hepatology. 2015.

23. Jung UJ, Torrejon C, Chang CL, Hamai H, Worgall TS, Deckelbaum RJ. Fatty acids regulate endothelial lipase and inflammatory markers in macrophages and in mouse aorta: a role for PPARgamma. Arterioscler Thromb Vasc Biol. 2012;32(12):2929-37.

24. Kosinski JR, Hubert J, Carrington PE, Chicchi GG, Mu J, Miller C, et al. The glucagon receptor is involved in mediating the body weight-lowering effects of oxyntomodulin. Obesity (Silver Spring). 2012;20(8):1566-71.

\section{Submit your next manuscript to BioMed Central and take full advantage of:}

- Convenient online submission

- Thorough peer review

- No space constraints or color figure charges

- Immediate publication on acceptance

- Inclusion in PubMed, CAS, Scopus and Google Scholar

- Research which is freely available for redistribution 